\title{
A COMPARISON OF CENTRALITY MEASURES IN SUSTAINABLE
}

\section{DEVELOPMENT GOALS}

\author{
Sena Ariesandy ${ }^{1}$, Ema Carnia², Herlina Napitupulu ${ }^{3 *}$ \\ 1,2,3 Department of Mathematics, Faculty of Mathematics and Natural Sciences, Universitas Padjadjaran \\ Jln. Raya Bandung Sumedang km 21 Jatinanagor, Sumedang, 45363, Indonesia, \\ e-mail: ${ }^{1}$ sena16002@mail.unpad.ac.id; 2ema.carnia@unpad.ac.id; 3*herlina@unpad.ac.id \\ Corresponding author*
}

\begin{abstract}
The Millennium Development Goals (MDGs), which began in 2000 with 8 goal points, have not been able to solve the global problems. The MDGs were developed into Sustainable Development Goals (SDGs) in 2015 with 17 targeted goal points achieved in 2030. Until now, methods for determining the priority of SDGs are still attractive to researchers. Centrality measure is one of the tools in determining the priority goal points on a network by using graph theory. There are four measurements of centrality used in this paper, namely degree centrality, betweenness centrality, closeness centrality, and eigenvector centrality. The calculation results obtained from the four measurements are compared dan analyzed, to conclude which goal points are the most prior and the least prior. Furthemore, in this paper we present other example with simple graph to show that each different centrality calculation possibly resulted different priority node, the calculation of this illustration is done using a Python's library named NetworkX.
\end{abstract}

Keywords: Sustainable Development Goals, Centrality Measures, Degree Centrality, Betweenness Centrality, Closeness Centrality, Eigenvector Centrality, Centralization 


\section{INTRODUCTION}

Sustainable Development Goals (SDGs), known as Global Goals, are sustainable development with objective to solve global problems such as overcoming environmental, political, and economics problems. In September 2015, the SDGs was agreed at a general meeting of the United Nations with 17 goals consisting of 169 targets, as a continuation from the 8 goals of MDGs.

In these 17 SDGs goal can be interpreted as goal that most influence other related goals. The reason of finding these priority goal points as a must is because of the limited funds, resources, and time that affect its completion. Social Network Analysis can be interpreted as a study about a relationship between variables (both economics, social, environmental and institutional variables) using graph theory [8]. Then, by examine the relationships structure between these variables in a group, may reveal how much the relationship between these variables occurs. In Social Network Analysis, the goal points of SDGs and their links are represented in a form of graphs. Goal points are represented by nodes while relationships between goal points are represented by edges. According to Blanc ([1]), a relationship of the goal points is the relationship of targets related to an existing goal points. For example, the target point from Goal 1, namely poverty, is related to the target point from Goal 2, namely hunger, then there is an interlinkage between that goal points. The SDGs graph representation used in this research is a weighted graph taken from ([1]) which means interlinkages between one goal point and another contained magnitude.

Research on centrality measures was introduced by Freeman ([5]) in his paper entitled "Centrality in Social Networks Conceptual Clarification". In 2008, Cadini ([3]) in a paper entitled "Using Centrality Measures to Rank the Importance of a Complex Network Infrastructure" discussed the calculation of centrality measures in power network systems. In implementing the SDGs, the application of centrality measures has been examined by Zhou and Moinuddin ([9]) for some country. Blanc in [1] studying and resulting the linkages/ links between the goal points alone for the global scope. In this paper we discussed in detail the application of centrality measures (that commonly applied in social network) based on the SDGs interlinkages produced in Blanc's research. In addition, authors want to point out that each centrality could show different characters, so that it could represent the centrality of different aspects.

\section{METHOD}

In this section, the theory of graph, centrality measures, eigenvectors and centralizations are explained in details.

These methods are very broad in use in the study of network analysis, and because the aim of this paper is to look at various aspects of centrality, these four uses of centrality are very appropriate. This method shows the character of each measurement from graph theory point of view. An explanation of the characteristics of each measurement is given in the discussion section.

\subsection{Graph ([7])}

A graph $G=(V, E)$ consist of $V$, a nonempty set of nodes (or vertices) and $E$, a set of edges. Each Edge has either one or two nodes associated with it, called its endpoints. An edge is said to connect its endpoints.

\subsection{Centrality}

Centrality measurement was firstly developed in Social Network Analysis (SNA). Research on centrality measures is currently also being done in determining priority goal points on SDGs. The relationship between goal points of Sustainable Development Goals is represented in the graph and then its centrality is calculated to see which goals are the most priority. The meaning of priorities in SDG's can be seen from various points of view depending on the objectives to be achieved. Therefore, different centrality calculations are performed in this research namely degree centrality, betweenness centrality, closeness centrality, and eigenvector centrality. The calculation of degree centrality and eigenvector centrality focus more on the degree of each node without regard to the weight of the graph while betweenness centrality and closeness centrality focus more to the calculation of the shortest path.

\subsubsection{Degree Centrality ([4])}

Degree centrality is used in estimating the importance of a direct relationship between a node and other 
node in the network. A formula in calculating the degree of centrality, $C_{D}(v)$, is given by:

$$
C_{D}(v)=\frac{d(v)}{n-1}
$$

where $d(v)$ is the number of degrees in a graph or the number of interactions that node $v$ has with other nodes in a network and $n$ is the number of nodes in the network.

\subsubsection{Betweenness Centrality ([4])}

Betweenness centrality calculates how often a node is passed by another node to go to a particular node in the network. The Dijkstra algorithm is used in determining the shortest path. The formula in calculating betweenness centrality, $C_{B}(v)$, is:

$$
C_{B}(v)=\frac{2}{(n-1)(n-2)} \sum_{s \neq t \neq v \in V} \frac{\sigma_{s t}(v)}{\sigma_{s t}}
$$

Where $\sigma_{s t}$ is the number of shortest paths from node $s$ to $t$ and $\sigma_{s t}(v)$ the number of shortest paths from node $s$ to $t$ passing through node $v$.

\subsubsection{Closeness Centrality ([4])}

Closeness centrality is used to calculate the average distance between a node and all other nodes in the network or in other words measure the closeness of a node with other nodes either directly or indirectly. The formula for calculating closeness centrality is:

$$
C_{C}(v)=\frac{n-1}{\sum_{t \in V} d(v, t)}
$$

where $d(v, t)$ is a shortest path distance from node $v$ to node $t$ and $n$ is a number of nodes on the network.

In betweenness centrality and closeness centrality, the shortest path is used in the calculation of the node. In this study, the Dijkstra algorithm is used in determining the shortest path. This algorithm is one of the simple methods that provides optimum value in searching the shortest path ([2]). The name of the Dijkstra algorithm is derived from its inventor, Edsger Dijkstra.

\subsubsection{Eigenvector Centrality ([10])}

Eigenvector centrality performs measurements that give higher weight to nodes that are connected to other nodes and also have high centrality values. Let the adjacency matrix $A$ contain entries in the form of $A_{v, t}$ and $\mathbf{x}$ is the eigenvector with the dominant eigenvalue $\lambda$. The eigenvector point centrality of a node $v$, namely $x_{v}$, can be calculated by the following formula:

$$
C_{E}(v):=x_{v}=\frac{1}{\lambda} \sum_{t \neq v \in V} A_{v, t} x_{t}
$$

In determining the dominant eigenvector of adjacency matrix $A$, a method so called Power Iteration is used simplify the calculation. The detail explanation is given in the following section.

\subsection{Power Iteration ([6])}

Power iteration, also known as power method, is an algorithm for calculating eigenvalues. For example, given a matrix $A$ that can be diagonalized, the algorithm will produce eigenvector that correspond to dominant eigenvalue $\lambda$ which is the largest eigenvalue (in absolute value) of matrix $A$, so that $A \mathbf{x}=\lambda \mathbf{x}$.

The algorithm of power iteration starts with the initialization of vector $\mathbf{b}_{0}$ which is an estimate of initial calculation of the dominant eigenvector. This method is explained as:

$$
\mathbf{b}_{k+1}=\frac{A \mathbf{b}_{k}}{\left\|A \mathbf{b}_{k}\right\|}
$$

where $\left\|A \mathbf{b}_{k}\right\|$ is the norm value of the product of the matrix $A$ with the vector $\mathbf{b}_{k}$ in the $k$-th iteration. The iterations will continue until $\left|\left\|A \mathbf{b}_{k+1}\right\|-\left\|A \mathbf{b}_{k}\right\|\right|<\epsilon$, where $\epsilon$ is a very small real number. The obtained $\mathbf{b}_{k+1}$ is taken as an eigenvector centrality where its point centrality $C_{E}(v)$ is the component of the eigenvector $\mathbf{b}_{k+1}$. 


\subsection{Centralization}

In addition, this paper will also calculate the centralization value of degree centrality, betweenness centrality, closeness centrality, and eigenvector centrality. Centralization of a network is a calculation of how central the most central node, in relation to the centrality value of other nodes ([5]). Centralization is calculated using the following formula:

$$
S^{2}=\frac{\sum_{i=1}^{n}\left[C_{x}\left(v_{i}\right)-\bar{C}_{x}\right]^{2}}{n}
$$

where $x$ is the type of centrality, $n$ is the number of nodes in the graph, $C_{x}\left(v_{i}\right)$ is centrality measure value at each node $v_{i}$, and $\bar{C}_{x}$ is the average value of centrality measures.

\section{RESULTS AND DISCUSSION}

In this research, the graph representation of Sustainable Development Goals is taken from the deep research of SDGs linkages by Blanc written in paper entitled "Towards integration at last? Sustainable development goals as a target network" ([1]). The graph is redrawn as can be seen in Figure 1.

The graph formed from Blanc is a representation of the graph SDGS where the goal points are represented by the nodes, and the relationship between the goal points is represented by the edges of graph. Weights in graph linkages of Fig. 1 indicate the number of targets linking different goals. For example, SDG peaceful and inclusive societies (no. 16) is linked with SDG on gender (no. 5) through four targets. If there is no target on one goal point related to the other end point, there is no edge connecting the goal point. The circles representing the goals on climate, land, energy and water have been singled out for purposes of comparison with other mappings in that paper. In this paper our purpose is to determine which goal points are more prior by measuring the degree centrality, betweenness centrality, closeness centrality, and eigenvector centrality of each goal point. The results and discussion are given in detail in this section.

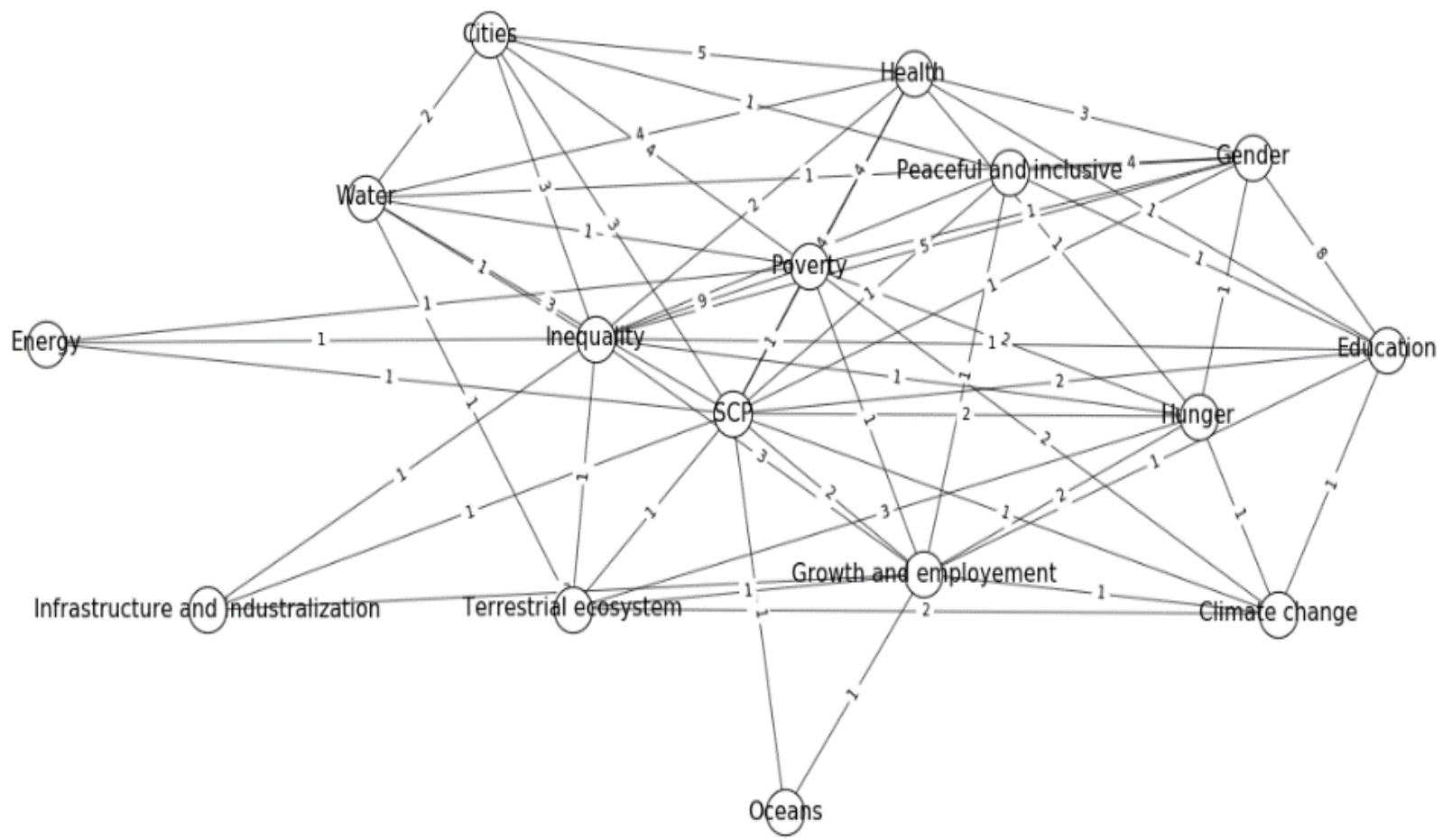

Figure 1. Graph representation of SDGs linkages taken from [1]

\subsection{Degree Centrality}

The value of degree centrality at each node is presented in Table 1 . Based on the results of the data in Table 1 , it is found that the largest degree centrality value is at the $12^{\text {th }}$ goal point, namely Responsible Consumption and Production followed by the $10^{\text {th }}$ goal point, namely Reduced Inequalities, while the 
lowest degree centrality values are at the $14^{\text {th }}$ goal point namely Life Below Water and $7^{\text {th }}$ and $9^{\text {th }}$ goal points in a row namely Affordable and Clean energy and Industry, Innovation, and Infrastructure. That is, when viewed on the Sustainable Development Goals, the $12^{\text {th }}$ point has a high interaction with other goal points or in other words, the Responsible Consumption and Production has the most part goal points that are related among the other goal points. Following is the example of the calculation of degree centrality of node 12 .

$$
C_{D}(12)=\frac{d(12)}{n-1}=\frac{14}{16-1}=0.9333333
$$

The value of degree centralization is obtained as follows,

$$
S^{2}=\frac{\sum_{i=1}^{n}\left[C_{D}\left(v_{i}\right)-\bar{C}_{D}\right]^{2}}{n}=0.0430556
$$

Table 1. Degree Centrality Measure

\begin{tabular}{|clc|clr|}
\hline $\begin{array}{c}\text { Nodes } \\
\left(\boldsymbol{v}_{\boldsymbol{i}}\right)\end{array}$ & \multicolumn{1}{c}{ Goal Points } & $\boldsymbol{C}_{\boldsymbol{D}}\left(\boldsymbol{v}_{\boldsymbol{i}}\right)$ & $\begin{array}{c}\text { Nodes } \\
\left(\boldsymbol{v}_{\boldsymbol{i}}\right)\end{array}$ & \multicolumn{1}{c|}{ Goal Points } & \multicolumn{1}{c|}{$\boldsymbol{C}_{\boldsymbol{D}}\left(\boldsymbol{v}_{\boldsymbol{i}}\right)$} \\
\hline 1 & Poverty & 0.6666667 & 9 & Infrastructure and industralization & $0.2000000^{(15)}$ \\
2 & Hunger & 0.5333333 & 10 & Inequality & $0.8000000^{(2)}$ \\
3 & Health & 0.5333333 & 11 & Cities & 0.4000000 \\
4 & Education & 0.46666667 & 12 & SCP & $0.9333333^{(\boldsymbol{I})}$ \\
5 & Gender & 0.5333333 & 13 & Climate change & 0.4000000 \\
6 & Water & 0.4666667 & 14 & Oceans & $0.1333333^{(\text {I6) }}$ \\
7 & Energy & $0.2000000^{(15)}$ & 15 & Terrestrial ecosystem & 0.4000000 \\
8 & Growth and employement & 0.6666667 & 16 & Peaceful and inclusive & 0.4000000 \\
\hline \hline
\end{tabular}

\subsection{Betweenness Centrality}

The value of betweenness centrality at each node is presented in Table 2. Based on the results in Table 2, it the greatest value of betweenness centrality is in the $12^{\text {th }}$ goal point, Responsible Consumption, followed by the $10^{\text {th }}$ goal point, Reduced Inequalities, while the lowest betweenness centrality values are at the $11^{\text {th }}$ goal point, Sustainable Cities and Communities, and $14^{\text {th }}$ goal point, Life Below Water. We can interpret that the $12^{\text {th }}$ goal point becomes a very important intermediary bridge among other goal points. In other words, if the goal points are related to the other goal points the problem will be solved, then the $12^{\text {th }}$ goal points that become the intermediary bridge also solved. Following is the example of the calculation of betweenness centrality of node 12 .

$$
\begin{aligned}
C_{B}(12) & =\frac{2}{(n-1)(n-2)} \sum_{s \neq t \neq v \in V} \frac{\sigma_{s t}(12)}{\sigma_{s t}} \\
& =\frac{2}{15 \cdot 14}\left(1+\frac{1}{3}+\frac{1}{3}++\frac{1}{2}+\cdots+\frac{1}{2}+\frac{1}{2}\right)=\frac{39349}{161700}=0.2433457
\end{aligned}
$$

The value of betweenness centralization is obtained as follows,

$$
S^{2}=\frac{\sum_{i=1}^{n}\left[C_{B}\left(v_{i}\right)-\bar{C}_{B}\right]^{2}}{n}=0.0041692
$$

Table 2. Betweenness Centrality for each node $v$

\begin{tabular}{|clc|clr|}
\hline \hline $\begin{array}{c}\text { Nodes } \\
(\boldsymbol{v})\end{array}$ & \multicolumn{1}{c|}{ Goal Points } & $\boldsymbol{C}_{\boldsymbol{B}}^{\prime}(\boldsymbol{v})$ & $\begin{array}{c}\text { Nodes } \\
(\boldsymbol{v})\end{array}$ & \multicolumn{1}{c|}{ Goal Points } & \multicolumn{1}{c|}{$\boldsymbol{C}_{\boldsymbol{B}}^{\prime}(\boldsymbol{v})$} \\
\hline 1 & Poverty & 0.0497317 & 9 & Infrastructure and industralization & 0.0050794 \\
2 & Hunger & 0.0618254 & 10 & Inequality & $0.1432966^{(2)}$ \\
3 & Health & 0.0064214 & 11 & Cities & $0.0013605^{(16)}$ \\
4 & Education & 0.1074765 & 12 & SCP & $0.2433457^{(1)}$ \\
5 & Gender & 0.0330128 & 13 & Climate change & 0.0324651 \\
6 & Water & 0.0346681 & 14 & Oceans & $0.0026455^{(15)}$ \\
7 & Energy & 0.0098413 & 15 & Terrestrial ecosystem & 0.0327740 \\
8 & Growth and employement & 0.1158019 & 16 & Peaceful and inclusive & 0.1123497 \\
\hline \hline
\end{tabular}




\subsection{Closeness Centrality}

The value of closeness centrality at each node is presented in Table 3. Based on the results of the data in Table 3, it is found that the greatest value of closeness centrality is in the $12^{\text {th }}$ goal point, which is Responsible Consumption, followed by the $8^{\text {th }}$ goal point, which is Decent Work and Economic Growth, while the lowest closeness centrality values are at the $11^{\text {th }}$ goal point, which is Sustainable Cities and Communities, and $3^{\text {th }}$ goal point, which is Good Health and Well-Being. It means, the $12^{\text {th }}$ goal point becomes a very important intermediary bridge among other goal points. In other words, if the goal points are related to the other goal points and the problem will be solved, then the $12^{\text {th }}$ goal points that become the intermediary bridge also solved. Following is the example of the calculation of closeness centrality of node 12.

$$
\boldsymbol{C}_{\boldsymbol{C}}(12)=\frac{\boldsymbol{n}-\mathbf{1}}{\sum_{t \in V} d(12, t)}=\frac{15}{13}=0.6521739
$$

The value of closeness centralization is obtained as follows

$$
S^{2}=\frac{\sum_{i=1}^{n}\left[C_{C}\left(v_{i}\right)-\bar{C}_{C}\right]^{2}}{n}=0.0044150
$$

Table 3. Closeness Centrality for each node $v$

\begin{tabular}{|clr|rlr|}
\hline \hline $\begin{array}{c}\text { Nodes } \\
(\boldsymbol{v})\end{array}$ & \multicolumn{1}{c|}{ Goal Points } & \multicolumn{1}{c|}{$\boldsymbol{C}_{\boldsymbol{C}}(\boldsymbol{v})$} & \multicolumn{1}{c|}{ Nodes } & \multicolumn{1}{c|}{ Goal Points } & \multicolumn{1}{c|}{$\boldsymbol{C}_{\boldsymbol{C}}(\boldsymbol{v})$} \\
\hline 1 & Poverty & 0.5555556 & 9 & Infrastructure and industralization & 0.4838710 \\
2 & Hunger & 0.5000000 & 10 & Inequality & 0.5769231 \\
3 & Health & $0.4166667^{(15)}$ & 11 & Cities & $0.3750000^{(16)}$ \\
4 & Education & 0.5769231 & 12 & SCP & $0.6521739^{(1)}$ \\
5 & Gender & 0.5357143 & 13 & Climate change & 0.5357143 \\
6 & Water & 0.5000000 & 14 & Oceans & 0.4545455 \\
7 & Energy & 0.5172414 & 15 & Terrestrial ecosystem & 0.5357143 \\
8 & Growth and employement & $0.6000000^{(2)}$ & 16 & Peaceful and inclusive & 0.5357143 \\
\hline
\end{tabular}

\subsection{Eigen vector Centrality}

The value of eigenvector centrality at each node is presented in Table 4. Based on the results of the data in Table 4, it is found that the greatest value of eigenvector centrality is in the $12^{\text {th }}$ goal point, which is Responsible Consumption, followed by the $10^{\text {th }}$ goal point, which is Reduced Inequalities, while the lowest closeness centrality values are at the $14^{\text {th }}$ goal point, which is Life Below Water, and $9^{\text {th }}$ goal point, which Industry, Inovation, and Infrastructure. It means, on the Sustainable Development Goals, Responsible Consumption and Production has extensive direct interaction with other goal points so that the Responsible Consumption and Production has a strategic position to connect the other goal points. Following is the example of the calculation of eigenvector point centrality of node 12. Following is the example of the calculation of eigenvector centrality using Power method in iteration $2^{\text {nd }}$ and $8^{\text {th }}$, this iteration converges in $9^{\text {th }}$ iteration, so that $C_{E}=\mathbf{b}_{\mathbf{9}}$.

- The $2^{\text {nd }}$ iteration:

$$
\begin{gathered}
|| \boldsymbol{A} \boldsymbol{b}_{2}\left\|=8.238907,||\left|\boldsymbol{A} \boldsymbol{b}_{2}\right|\left|-\left\|\boldsymbol{A} \boldsymbol{b}_{1}\right\|\right|=0.037135\right. \\
\mathbf{b}_{2}=[0.3167936,0.2858869,0.2781603,0.2472536 \\
0.2781603,0.2472536,0.1390801,0.2858870 \\
0.1390801,0.3167936,0.2202102,0.3477003 \\
0.2124835,0.0927201,0.2202102,0.2202102]
\end{gathered}
$$

- The $8^{\text {th }}$ iteration:

$$
\begin{gathered}
|| \boldsymbol{A} \boldsymbol{b}_{\mathbf{8}}\left\|=8.246703,||\left|\boldsymbol{A} \boldsymbol{b}_{\mathbf{8}}\|-\| \boldsymbol{A} \boldsymbol{b}_{7} \|\right|=0.000001\right. \\
\mathbf{b}_{\mathbf{8}}=[0.3180555,0,2778104,0.2768045,0.2392692 \\
0,2703975,0.2436250,0.1252324,0.2882918 \\
0.1216475,0.3393867,0.2137566,0.3742264 \\
0.2070504,0.0804252,0.2099411,0.2100369]
\end{gathered}
$$


The value of closeness centralization is obtained as follows,

$$
S^{2}=\frac{\sum_{i=1}^{n}\left[C_{E}\left(v_{i}\right)-\bar{C}_{E}\right]^{2}}{n}=0.0060492
$$

Table 4. Eigenvector point Centrality for each node $v$

\begin{tabular}{|cll|lll|}
\hline $\begin{array}{c}\text { Nodes } \\
(\boldsymbol{v})\end{array}$ & \multicolumn{1}{c}{ Goal Points } & $\boldsymbol{C}_{\boldsymbol{E}}(\boldsymbol{v})$ & $\begin{array}{c}\text { Nodes } \\
(\boldsymbol{v})\end{array}$ & \multicolumn{1}{c|}{ Goal Points } & $\boldsymbol{C}_{\boldsymbol{E}}(\boldsymbol{v})$ \\
\hline 1 & Poverty & 0.3180284 & 9 & Infrastructure and industralization & $0.1215391^{(15)}$ \\
2 & Hunger & 0.2777375 & 10 & Inequality & $0.3395592^{(2)}$ \\
3 & Health & 0.2767879 & 11 & Cities & 0.2137019 \\
4 & Education & 0.2391863 & 12 & SCP & $0.3744126^{(1)}$ \\
5 & Gender & 0.2763845 & 13 & Climate change & 0.2070591 \\
6 & Water & 0.2435802 & 14 & Oceans & $0.0803640^{(16)}$ \\
7 & Energy & 0.1251408 & 15 & Terrestrial ecosystem & 0.2098625 \\
8 & Growth and employement & 0.2883242 & 16 & Peaceful and inclusive & 0.2099709 \\
\hline \hline
\end{tabular}

\subsection{Discussion}

Comparison of the four centrality measures in Table 5, shows that the most priority factor is at node $12^{\text {th }}$, which is Responsible Consumption and Production. This goal point should be the main focus in the global problems that faced by various countries in the world. Meanwhile, the goal point with small priorities are Sustainable Cities and Communities and Life Below Water. Calculated in terms of the degree centrality or direct relationship between the goal points, Life Below Water is the smallest value of priority. Whereas if calculated in terms of the shortest distance, Sustainable Cities and Communities has the smallest prior value. The comparison between all centrality results are given as well in the form of bar chart in Figure 2.

Table 5. Comparison of Centrality Measures

\begin{tabular}{|c|l|r|r|r|r|}
\hline Node & \multicolumn{1}{|c|}{ Goal points } & \multicolumn{1}{c}{$\begin{array}{c}\text { Degree } \\
\text { Centrality }\end{array}$} & $\begin{array}{c}\text { Betweenness } \\
\text { Centrality }\end{array}$ & \multicolumn{1}{c|}{$\begin{array}{c}\text { Closeness } \\
\text { Centrality }\end{array}$} & $\begin{array}{c}\text { Eigenvector } \\
\text { Centrality }\end{array}$ \\
\hline 1 & Poverty & 0.6666667 & 0.0497317 & 0.5555556 & 0.3180158 \\
\hline 2 & Hunger & 0.5333333 & 0.0618254 & 0.5000000 & 0.2777054 \\
\hline 3 & Health & 0.5333333 & 0.0064214 & 0.4166667 & 0.2767806 \\
\hline 4 & Education & 0.4666667 & 0.1074765 & 0.5769231 & 0.2391494 \\
\hline 5 & Gender & 0.5333333 & 0.0330128 & 0.5357143 & 0.2763789 \\
\hline 6 & Water & 0.4666667 & 0.0346681 & 0.5000000 & 0.2435601 \\
\hline 7 & Energy & 0.2000000 & 0.0098413 & 0.5172414 & 0.1251007 \\
\hline 8 & Growth and employment & 0.6666667 & 0.1158019 & 0.6000000 & 0.2883394 \\
\hline 9 & Infrastructure and industrialization & 0.2000000 & 0.0050794 & 0.4838710 & 0.1214916 \\
\hline 10 & Inequality & 0.8000000 & 0.1432966 & 0.5769231 & 0.3396349 \\
\hline 11 & Cities & 0.4000000 & $0.0013605 * *$ & $0.3750000 * *$ & 0.2136775 \\
\hline 12 & SCP & $\mathbf{0 . 9 3 3 3 3 3 3 *}$ & $\mathbf{0 . 2 4 3 3 4 5 7 *}$ & $\mathbf{0 . 6 5 2 1 7 3 9} *$ & $\mathbf{0 . 3 7 4 4 9 4 4 *}$ \\
\hline 13 & Climate change & 0.4000000 & 0.0324651 & 0.5357143 & 0.2070639 \\
\hline 14 & Oceans & $0.1333333 * *$ & 0.0026455 & 0.4545455 & 0.0803373 \\
\hline 15 & Terrestrial ecosystem & 0.4000000 & 0.0327740 & 0.5357143 & 0.2098282 \\
\hline 16 & Peaceful and inclusive & 0.4000000 & 0.1123497 & 0.5357143 & 0.2099419 \\
\hline & & 0.0430556 & 0.0041692 & 0.0044150 & $0.0060492 * *$ \\
\hline
\end{tabular}

Note: Value with asterisks (*) mean the greatest value in its centrality measures, meanwhile with double asterisks $(* *)$ are the smallest in its centrality measures. 


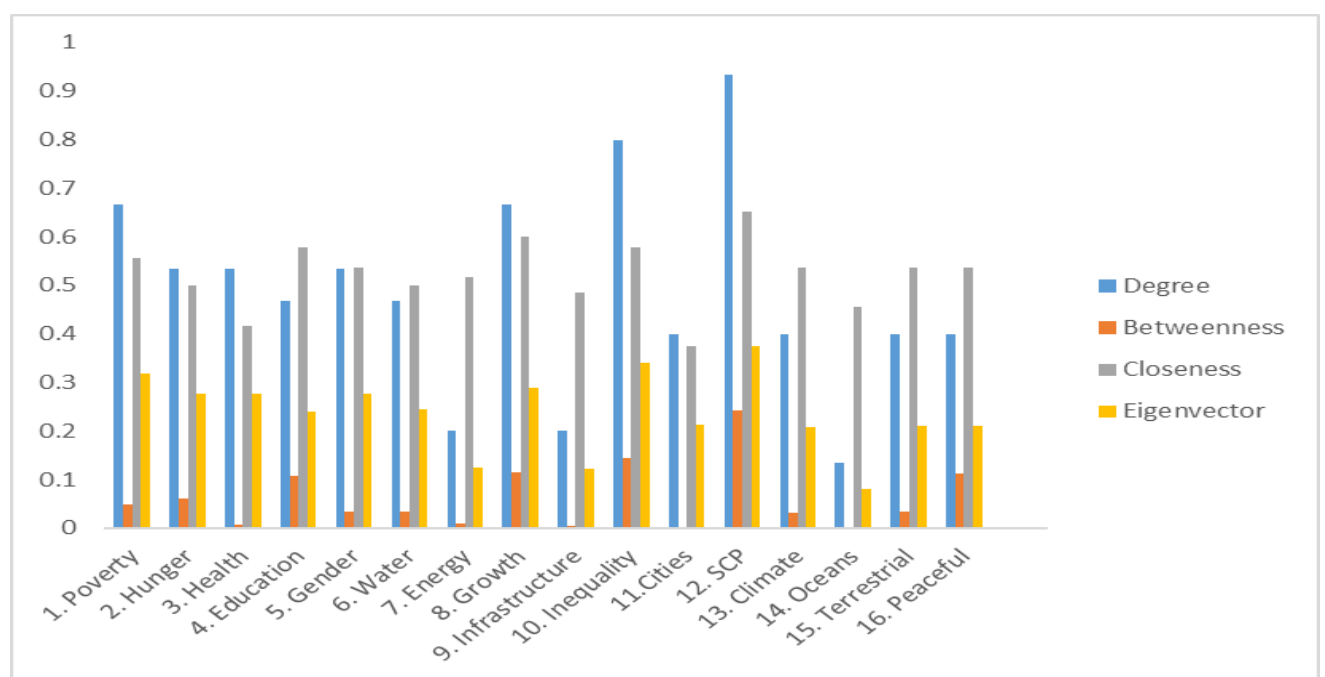

Figure 2. Comparison of Centrality Measures in Bar Chart

Visualization of the graph based on the four centrality calculations is given in Figure 3, where the size of the nodes in each figure is based on the size of each type of centrality

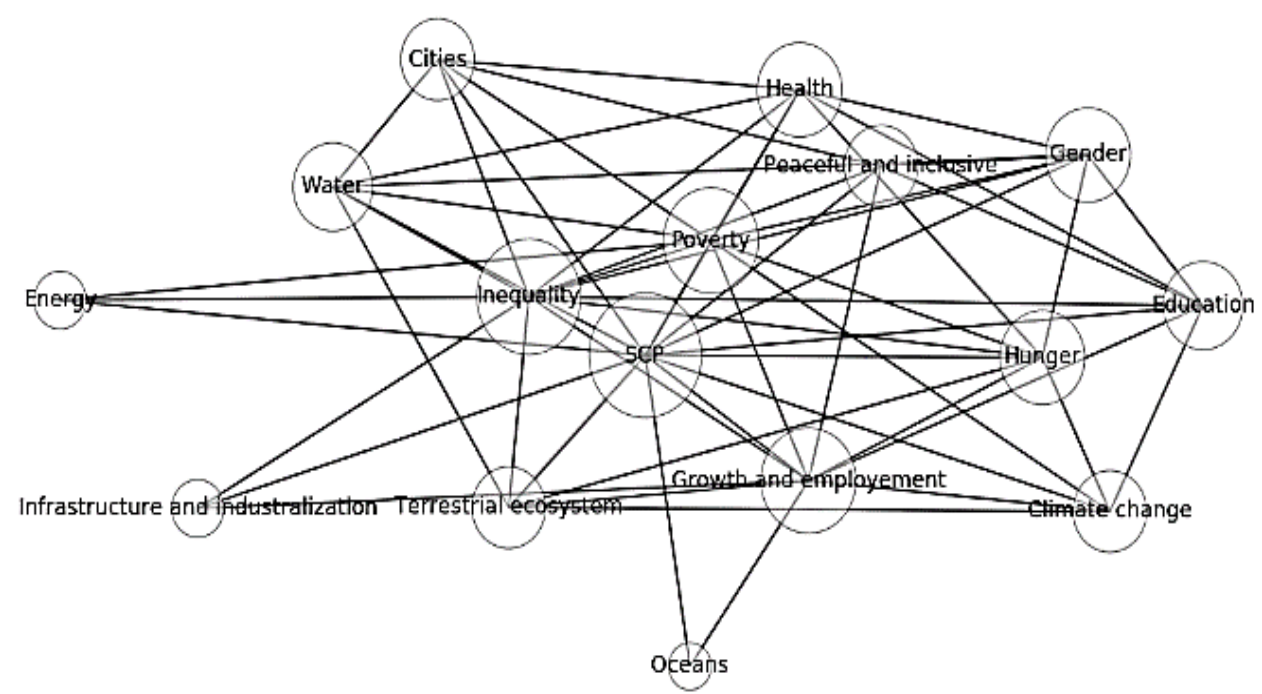

(a)

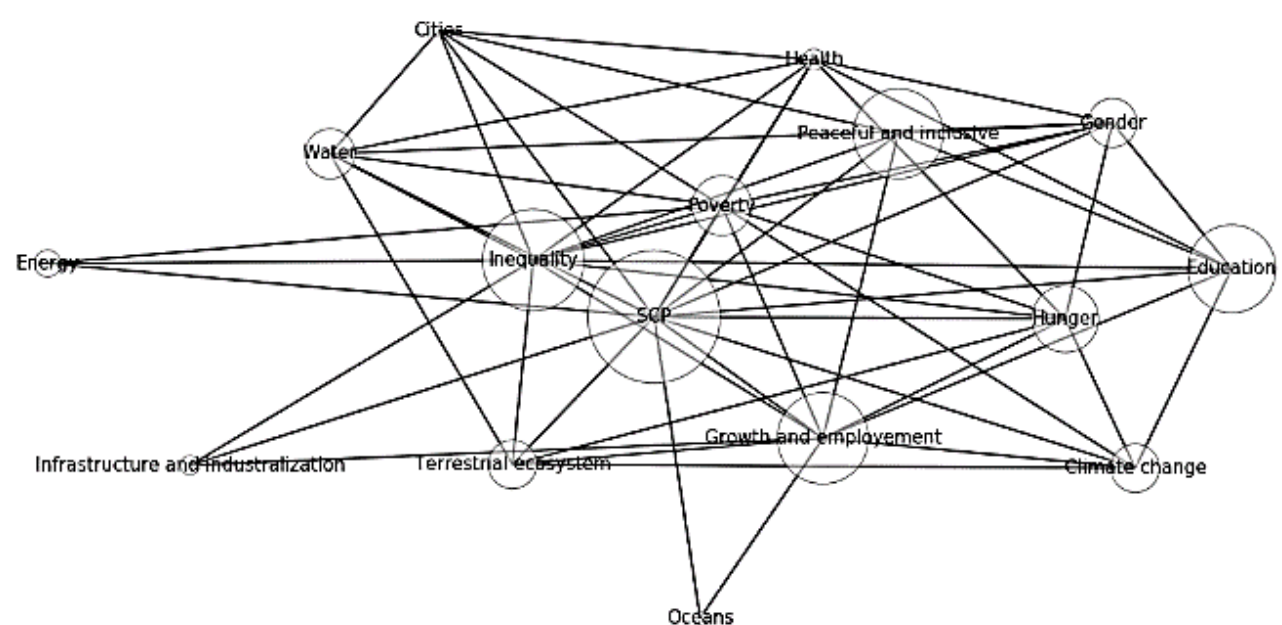

(b) 


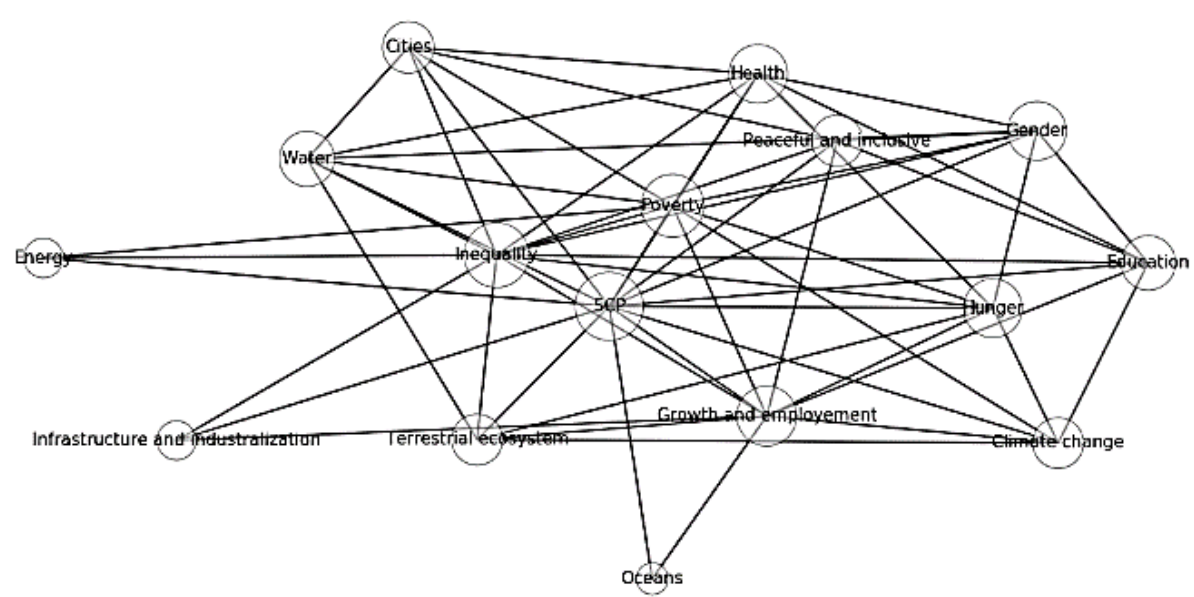

(c)

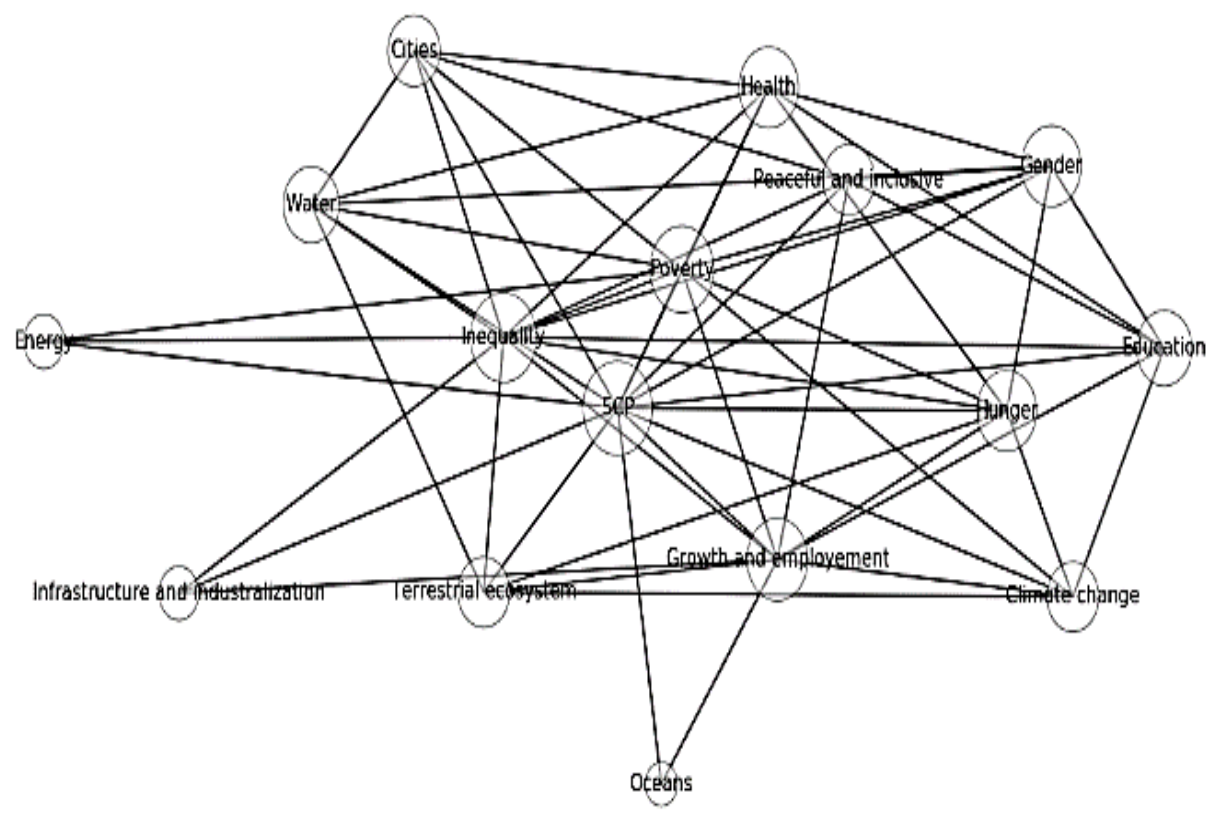

(d)

Figure 3. Plot of SDGs graph with size of nodes are proportional to Centrality measures: (a) Degree Centrality, (b) Betweenness Centrality, (c) Closeness Centrality, (d) Eigenvector Centrality measure

\subsubsection{Counter Example of priority node in each centrality}

In this section, we will explain through illustration of simple graph as shown in Figure 4, that another type of graph may resulted to different priority node for different centrality measures. The four centrality measures of graph in Figure 4 are calculated as seen in Table 6.

The calculation of centrality measures can be done using library NetworkX in software Python 3.7, we give the coding program below. Please note that the two dots in line 4 and 5 of coding below supposed to be filled with the complete nodes and edges of graph $G$ respectively, but for simplicity in this paper it is left only by dots. In this coding, since the weight of each edge of Graph Figure 4 is equal to 1 thus we didn't add a weight in edge. The value displays in Table 6 only 5 decimal places taken.

Based on the results on Table 4, it can be seen that centrality measures can be varied. For degree centrality node, the $2^{\text {nd }}$ and $11^{\text {th }}$ nodes have the greatest value. The priority on betweenness centrality and closeness centrality node is in point $1^{\text {st }}$. While the eigenvector centrality node priority is in $2^{\text {nd }}$ and $11^{\text {th }}$ node. This illustration shows for any graph probably resulted to different priority node in different centrality measures 


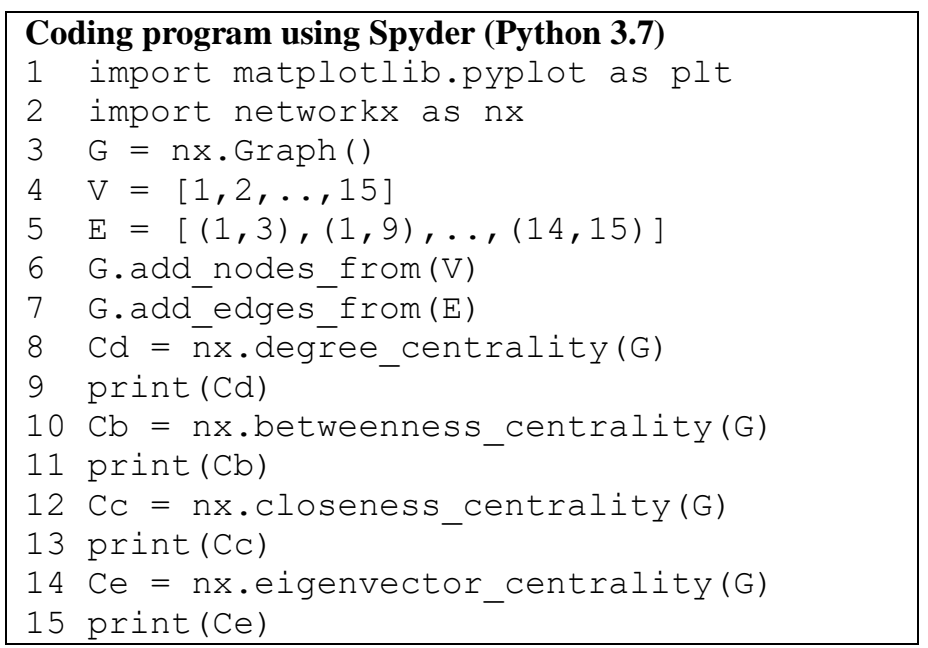

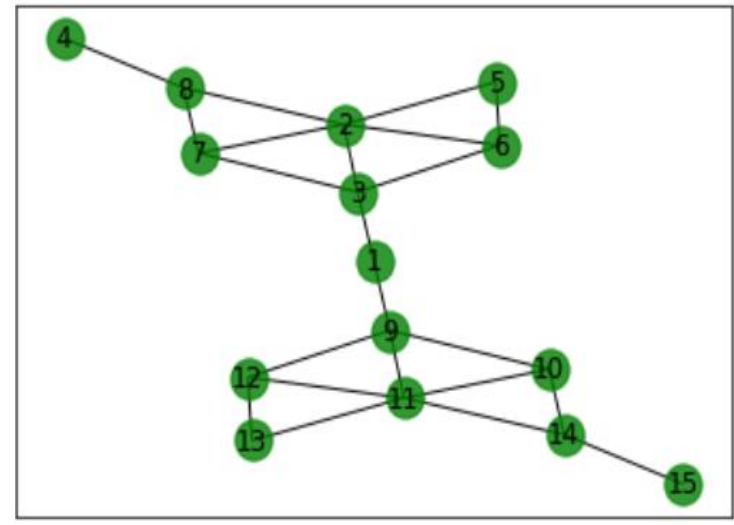

Figure 4. Graph for counterecample of centrality of priority node

Table 6. Centrality measures of each node of counterecample of priority node

\begin{tabular}{|r|r|r|r|r|}
\hline \multirow{2}{*}{ Node } & \multicolumn{4}{|c|}{ Centrality measures of each node } \\
\cline { 2 - 5 } & \multicolumn{1}{|c|}{ Degree } & Betweenness & Closeness & Eigenvector \\
\hline 1 & 0.14285 & $\mathbf{0 . 5 3 8 4 6}^{*}$ & $\mathbf{0 . 4 1 1 7 6}$ & 0.19565 \\
2 & $\mathbf{0 . 3 5 7 1 4} *$ & 0.20879 & 0.35000 & $\mathbf{0 . 3 7 6 9 4}$ \\
3 & 0.28571 & 0.53296 & 0.40000 & 0.32970 \\
4 & 0.07142 & 0.00000 & 0.22222 & 0.06267 \\
5 & 0.14285 & 0.00000 & 0.26923 & 0.19085 \\
6 & 0.21428 & 0.04945 & 0.32558 & 0.26629 \\
7 & 0.21428 & 0.09890 & 0.33333 & 0.27234 \\
8 & 0.21428 & 0.14285 & 0.28000 & 0.21124 \\
9 & 0.28571 & 0.53296 & 0.40000 & 0.32970 \\
10 & 0.21428 & 0.09890 & 0.33333 & 0.27234 \\
11 & $\mathbf{0 . 3 5 7 1 4} *$ & 0.20879 & 0.35000 & $\mathbf{0 . 3 7 6 9 4} *$ \\
12 & 0.21428 & 0.04945 & 0.32558 & 0.26629 \\
13 & 0.14285 & 0.00000 & 0.26923 & 0.19085 \\
14 & 0.21428 & 0.14285 & 0.28000 & 0.21124 \\
15 & 0.07142 & 0.00000 & 0.22222 & 0.06267 \\
\hline
\end{tabular}

\section{CONCLUSION}

Based on the calculation value of degree centrality, betweenness centrality, closeness centrality, and eigenvector centrality, it is obtained that the $12^{\text {th }}$ goal point, Responsible Consumption and Production has the greatest centrality value compared to other goal points. It means, the $12^{\text {th }}$ goal point is the most central goal point (for all measurements of centrality that has been done) so that it is considered as the most priority.

It can be seen that the $12^{\text {th }}$ goal point is Responsible Consumption and Production has the highest centrality value compared to other goal points. In addition, it can be concluded that the greatest value of centralization is value degree centralization. So that the node is central to the calculation. degree centralization is the most influential node in the network.

So, we get the result that the $12^{\text {th }}$ goal point, Responsible Consumption and Production is the most priority goal point. We quite sure that the method is good for problems that can be represented by linkages relations. Furthermore, in this paper the other example in the form of simple graph is explained that each different centrality measures type possibly resulted different priority node, instead one node only. 


\section{BIBLIOGRAPHY}

[1] D. L. Blanc, "Towards Integration at Last? The Sustainable Development Goals as a Networks of Targets", Sustainable Development, vol. 23, no. 3, pp. 176-187, May-Jun 2015.

[2] U. Brandes, "A Faster Algorithm for Betweenness Centrality", Journal of Mathematical Sociology, vol. 25, no. 2, pp. 163-177, 2001

[3] F. Cadini, E. Zio, C. A. Petrescu, "Using Centrality Measures to Rank the Importance of the Components of a Complex Network Infrastructure", In: Setola R., Geretshuber S. (eds). Critical Information Infrastructure Security. CRITIS 2008. Lecture Notes in Computer Science, vol. 5508, Berlin: Springer, 2008, pp. 155-167

[4] C. Donninger. "The distribution of centrality in social network". Social Networks, vol. 8, no.2, pp. 191-203, June 1986

[5] L.C. Freeman, "Centrality in Social Network Conceptual Clarification". Social Networks, vol. 1, issue 3, pp. 215-239, 1978-1979

[6] N. Meganathan, "Eigenvector Centrality-Based Mobile Target Tracking in Wireless Sensor Networks" In N. Kamila (Ed.) Handbook of Research on Wireless Sensor Network Trends, Technologies, and Applications., Hershey PA: IGI Global, 2017, pp. 395-412

[7] K. H. Rosen, Discrete Mathematics and Its Applications, $7^{\text {th }}$ Edition, New York: McGraw-Hill Companies, Inc., 2012

[8] M. Tsvetovat and A. Kouznetsov, Social Network Analysis for Startups, Sebastopol: O'Reilly Media, 2011

[9] X. Zhou and M. Moinuddin, "Sustainable Development Goals Interlinkages and Network Analysis: A Practical Tool for SDG Integration and Policy Coherence", Institute for Global Environmental Strategies (IGES) Research Report, 2017

[10] A. Lange, Centrality in Strategic Transportation Network Design, An application to less-than-truckload networks. Wiesbaden: Springer Gabler, 2019 
